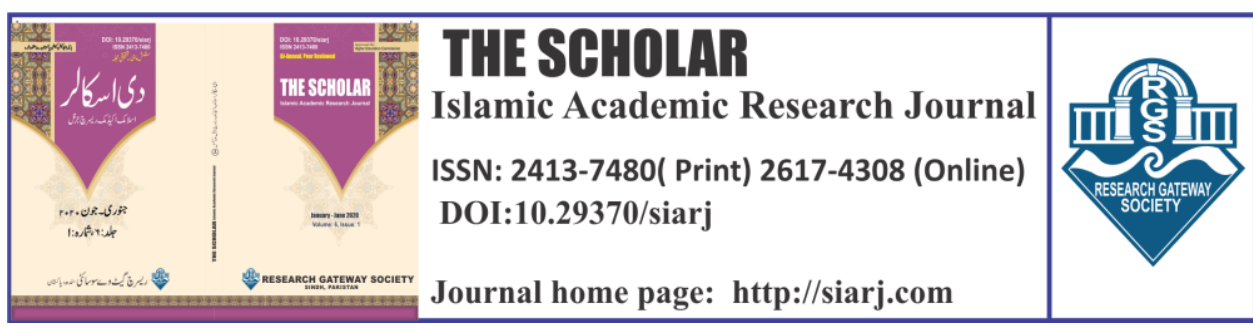

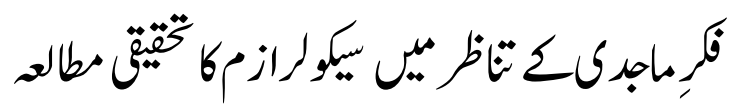

\title{
A RESEARCH STUDY OF SECULARISM IN CONTEXT OF MAJIDI THOUGHT
}

\section{Abdul Majid}

Ph.D Scholar, Department of Islamic Studies,

University of Engineering and Technology

Lahore, Pakistan

Email : profabughufran475@gmail.com

\section{ORCID ID:}

https://orcid.org/0000-0002-7122-1017

\section{Dr. Attiq ur Rehman}

Associate Professor, Department of Islamic

Studies, University of Engineering and

Technology Lahore, Pakistan

Email: dratiquet@gmail.com

\section{ORCID |D:}

https://orcid.org/0000-0003-2085-4110

To cite this article:

Majid, Abdul, and Attiq ur Rehman. "URDU- A RESEARCH STUDY OF

SECULARISM IN CONTEXT OF MAJIDI THOUGHT.” The Scholar-Islamic

Academic Research Journal 6, No. 1 (May 31, 2020): 41-55.

To link to this article: https://doi.org/10.29370/siarj/issue10ar5

Journal

Publisher

DOI:

URL:

License:

Journal homepage

Published online:
The Scholar Islamic Academic Research Journal

Vol. 6, No. 1 || January -June 2020 || P. 41-55

Research Gateway Society

10.29370/siarj/issue10ar5

https://doi.org/10.29370/siarj/issue10ar5

Copyright c 2017 NC-SA 4.0

www.siari.com

2019-05-31

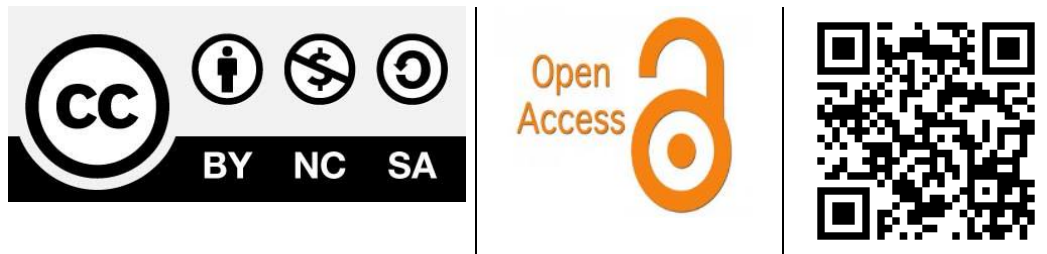


THE SCHOLAR (January - June 2020)

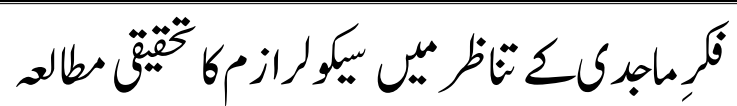

\title{
A RESEARCH STUDY OF SECULARISM IN CONTEXT OF MAJIDI THOUGHT
}

\author{
Abdul Majid, Dr. Attiq ur Rehman
}

\begin{abstract}
:
Secularism is a western term which means a complete separation of all political and other matters of the state from religion. Secularism is not against religion but it declares all the moral and revealed values as a private affair of a man's life. Maulana Abdul Majid Daryabadi, a renowned religious scholar, is not in favour of secular thoughts. He is of the view that Islam must have a supreme status. In his strong opinion, religion is the very breath of life, it matters above everything; it is the mainspring of a Muslim's conduct. A person with secular thoughts wants solution of every matter of the state according to human reason. All the political, economic and social issues should be solved in the light of human experiences. But Maulana strongly declares that creature must seek guidance from the Creator who knows His creation best. He says that Islam calls for the complete transformation of personality; and convert to Islam must be renewed in every corner of his being.
\end{abstract}

KEYWORDS: Abdul Majid Daryabadi, Western Thought,

Education, Civilization, State Affairs.

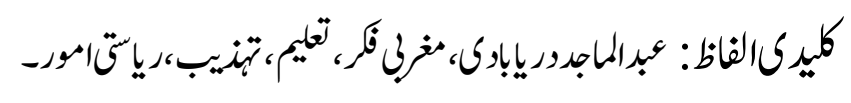




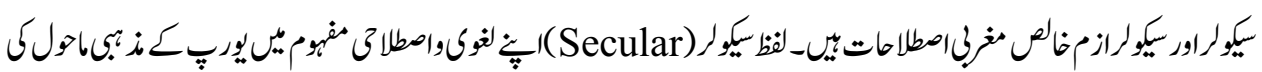

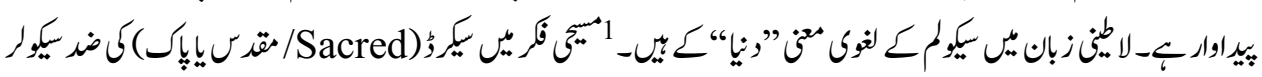
عecular) (Secular)

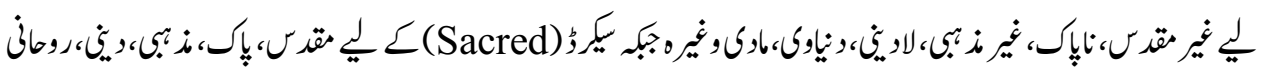
3.

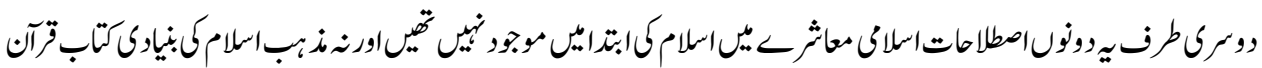

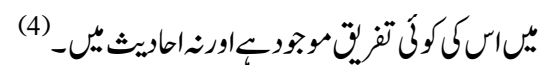

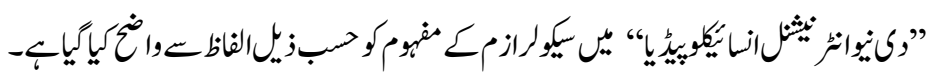

As its name implies, it eoncentrates its attention upon the present life, neither " denying nor affirming the existence of another. it inculcates an ethics not depent in any way on religion, although it does not formaly deny the truth of any riligion." 5

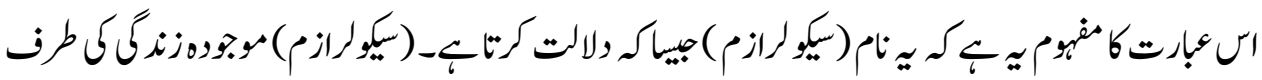

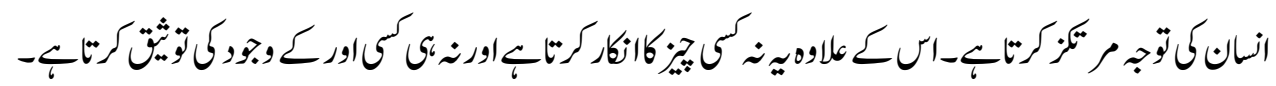

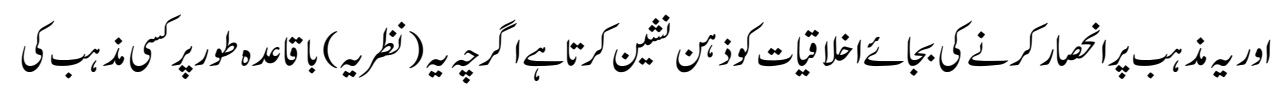

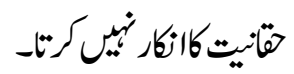
"انسائلويئيل

\footnotetext{
${ }^{1}$ Sibt-e-Hasan, Naveed e Fikr, Karachi: Maktaba Danial, 2002, p.69

2 Javed Iqbl, Dr, Afkar e lqbal, Tashrihat e Javed, Lahore: Sang e mail

Publications, 2005, p.121

${ }^{3}$ Fazl-ul-Rahman, Dr, Islam awr Jadidiyat, Tarjuma, MuhmmadKazim, Lahore: Masha'al, 1998,P 12; Afkar e lqbal, Tashrihat e Javed, p.10

${ }^{4}$ Manzur Ahmad, Dr., Islam Chand Fikri Masail, Lahore: Idarathaqafat-e-IsImiah, 2004, p.10

${ }^{5}$ The New International, p.750 vol 17. New york, Dodd mead and Company, 1907
} 
secularism is an ethical system founded on the principles of natural morality " and independent of revealed religion or super naturalism" ${ }^{6}$

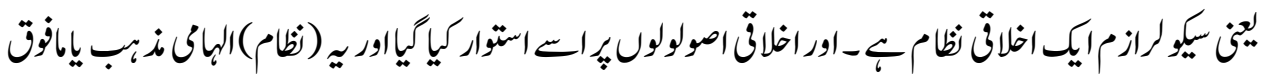

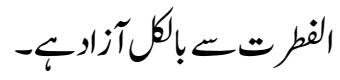

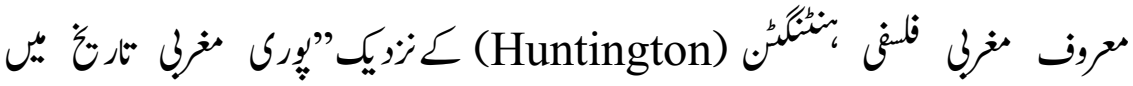

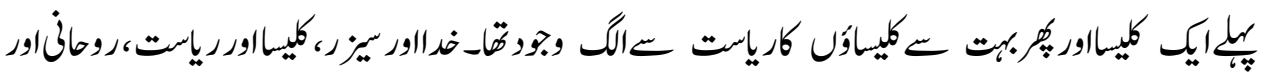

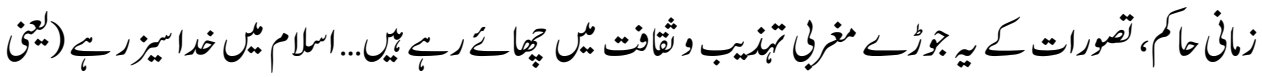

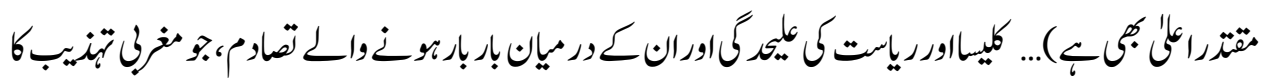

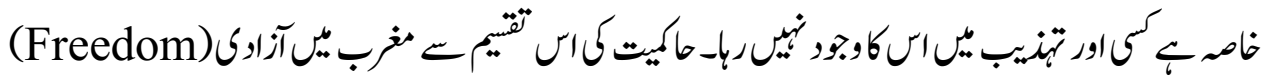

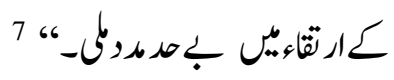

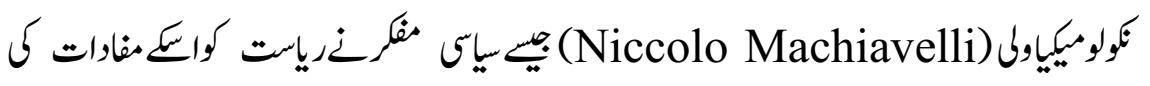

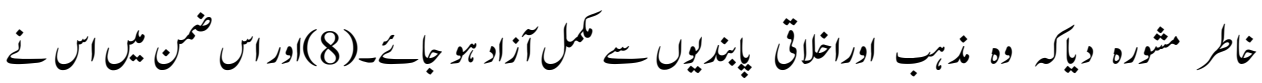

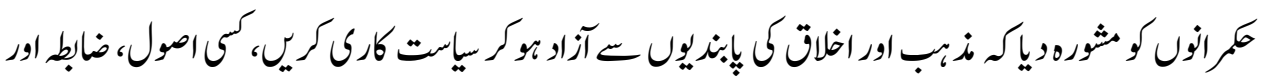

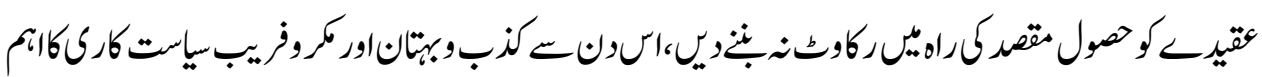

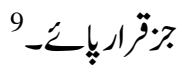

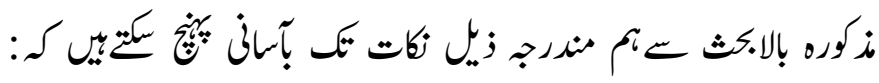

${ }^{6}$ Encyclopaedia Americana, p.510 vol. 24 New York, 1957

${ }^{7}$ Huntington, Samuel,P, Tehzibon ka Tasadum awr Aalmi Nizam ki Tashkil e Nao, Tarjuma: Suhail Anjum, Karachi, Oxford University Press, 2003, p.70

${ }^{8}$ Bronowski J. \& Bruce Mazlish, Western Intellectual Tradition, New York: Harper \&brothers publishers, 1960, p.43

${ }^{9}$ Muhammad Saleem, Sayyed, Maghrabi Falsafa e Taleem, Aik Tanqidi Mutala'a, Karachi: Zawar Academy Publications, 2008, p.48 


$$
\begin{aligned}
& \text { ） i } \\
& \text { ） （ii } \\
& \text { (iii }
\end{aligned}
$$

：iv

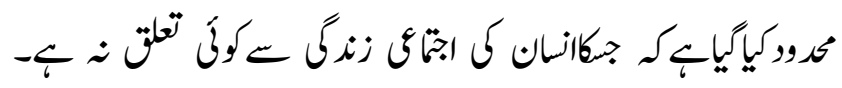

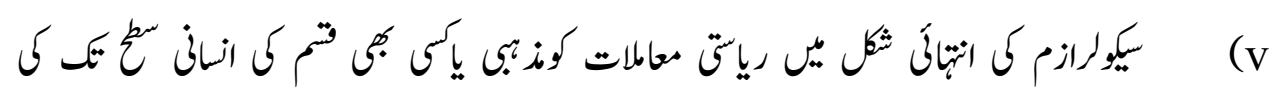
اخلاقيات سع آزاوكروياكياب -

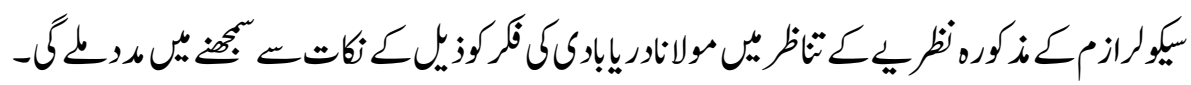

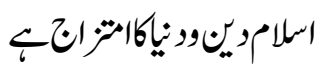

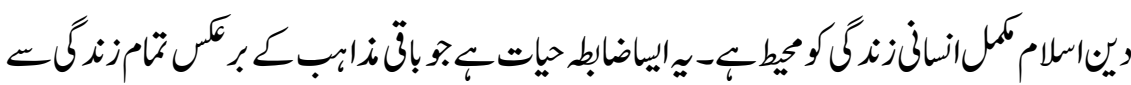

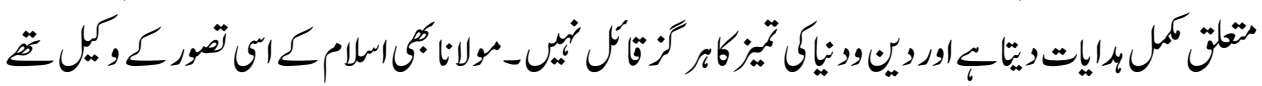

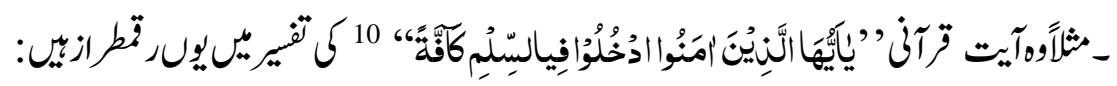

The secular and the religious, the material and the spiritual are not separated " in the all-inclusive system of Islam. Whatever may be true of other faiths and creeds, in Islam religion is the very breath of life, it matters above everything; it is the mainspring of a Muslim's conduct. ${ }^{11}$

${ }^{10}$ Al-Baqrah: 208

11 Tafsir-e-Majidi, Vol I, P II, n. 362 


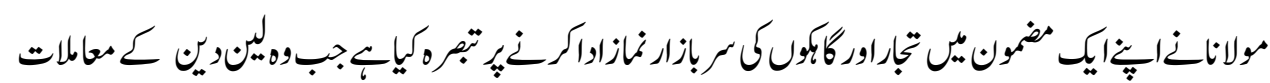

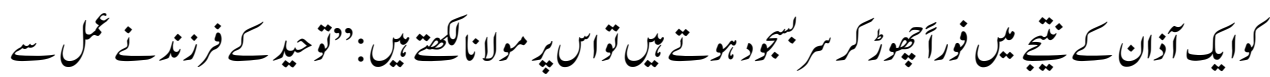

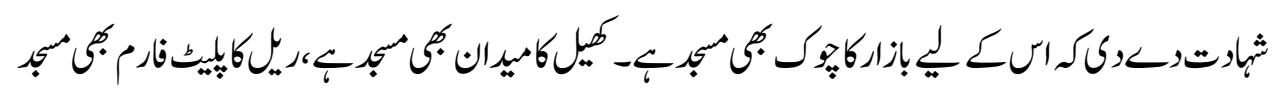

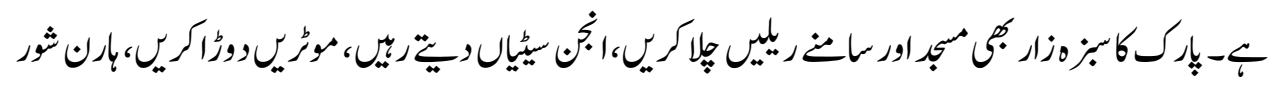

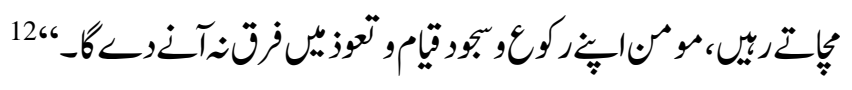

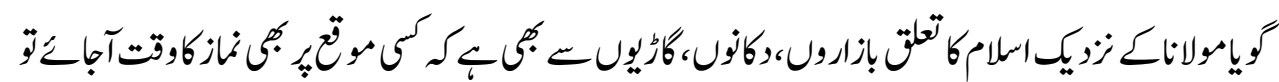

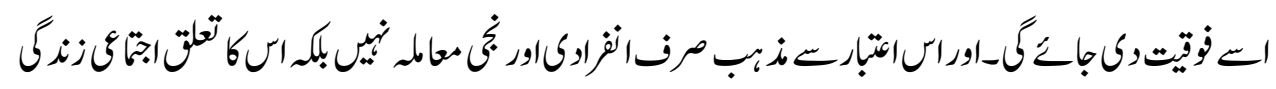

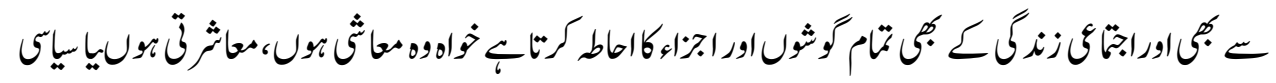

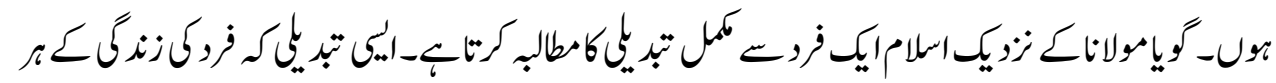

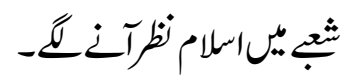

\section{رياتثامور،اسلام اور مولاناوببرالماجدوريابارك:}

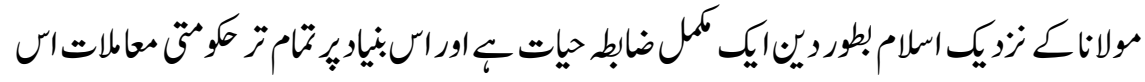

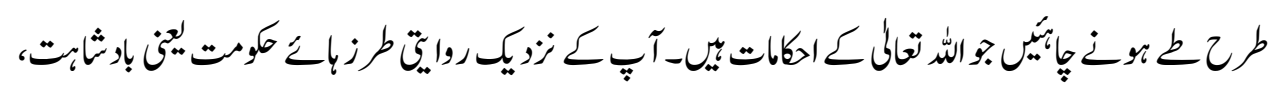

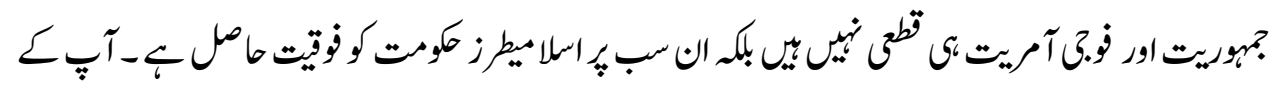

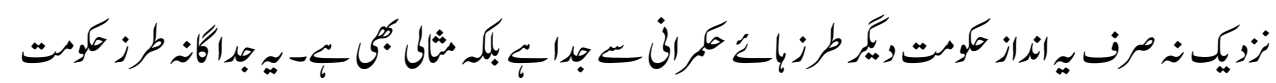

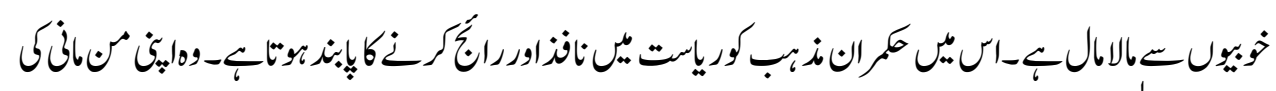

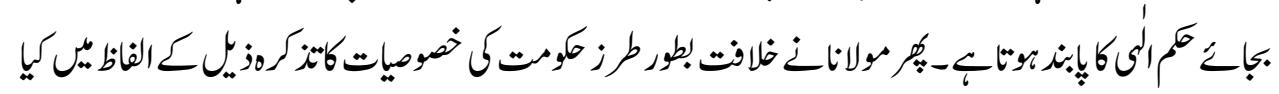

12 Daryabadi, Abdul Majid, Mawlana, Millat e Islamia awr asr e hazir k taqazay, Murattab: Muhammad Musa Bhutto, Haidar Abad: Sindh National Academy, 2006, p.127 


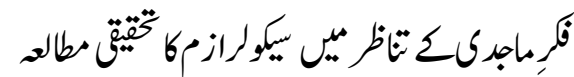

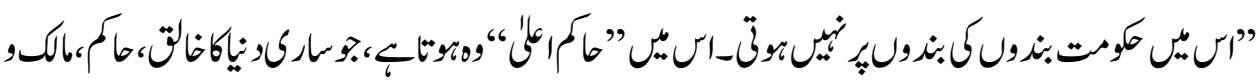

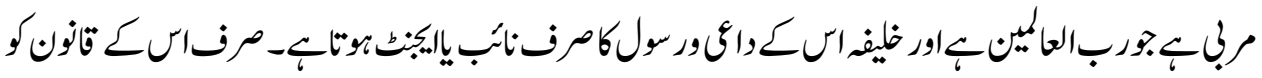

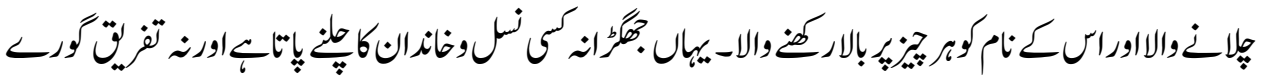

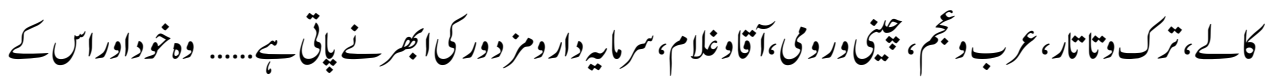

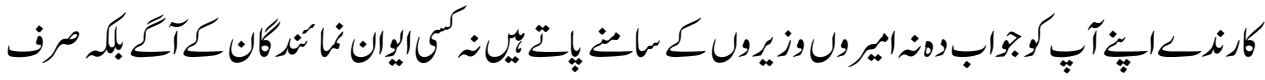

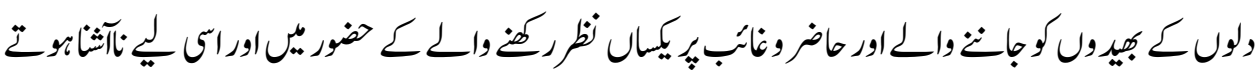

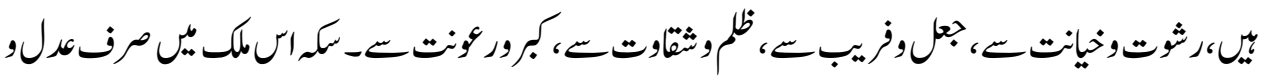

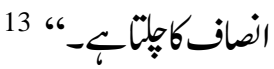

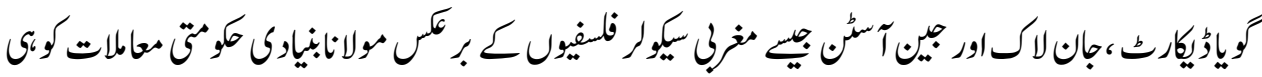

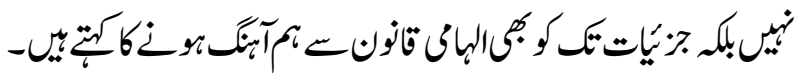

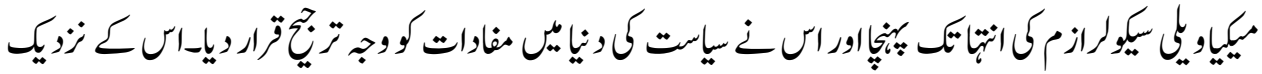

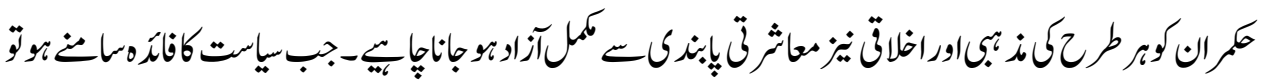

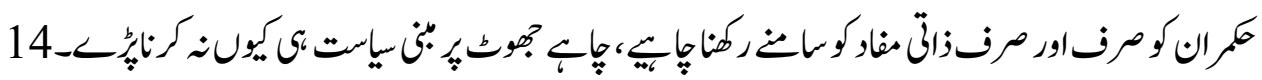

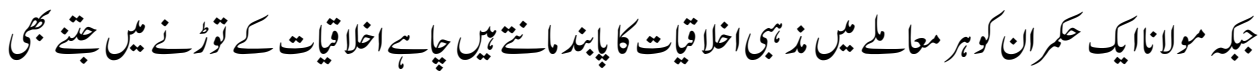

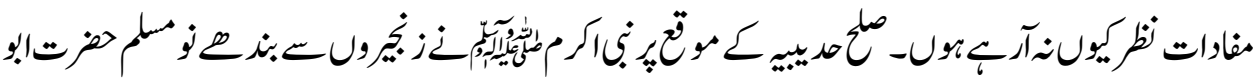

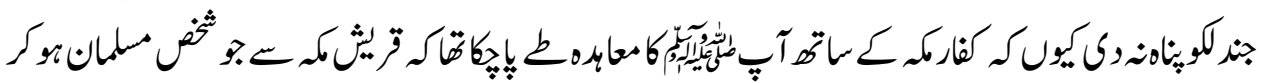

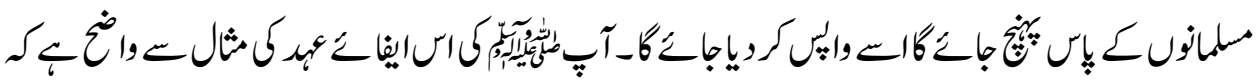

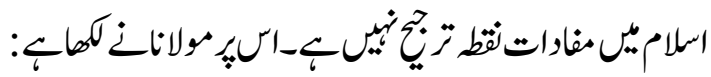

13 "Sachi Baten", Maulana Abdul Majid Daryabadi, Haftawar Siddique Jadid, Lakhnau, Jild9 Shumara 5, 2 January 1959, P1

14 Thomson, Devid, (Ed.), Political Ideas, London: Penguin Books, 1977, P 28 


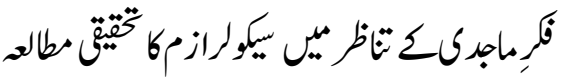

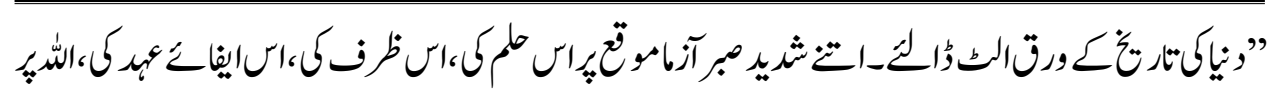

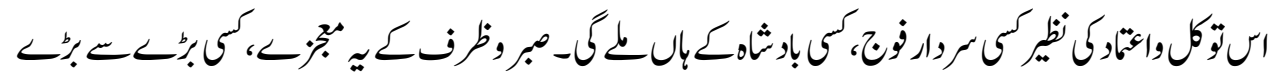
15 ارى 15

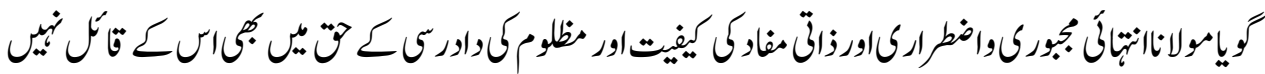

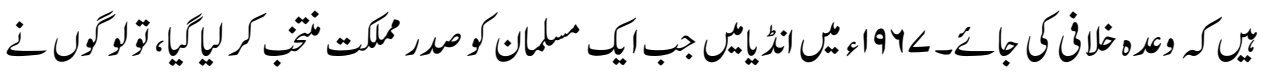

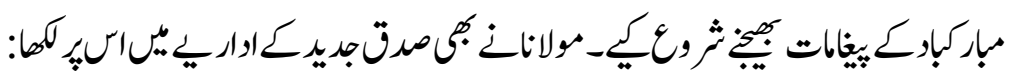

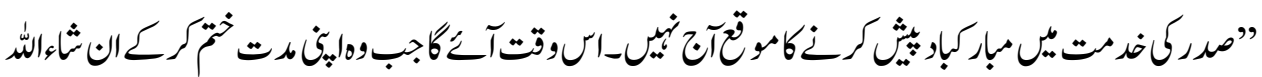

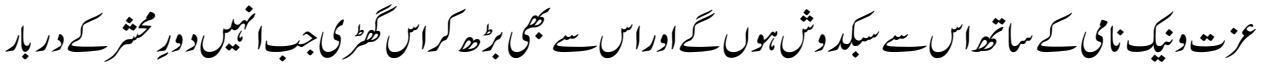

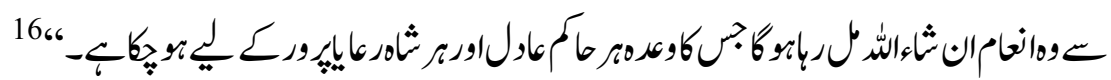

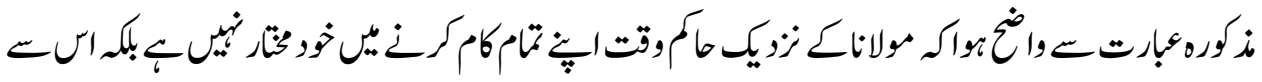

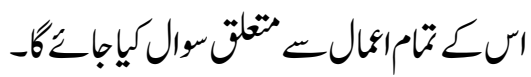

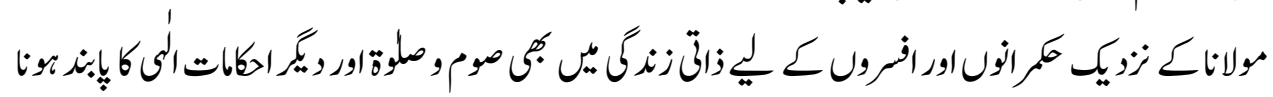

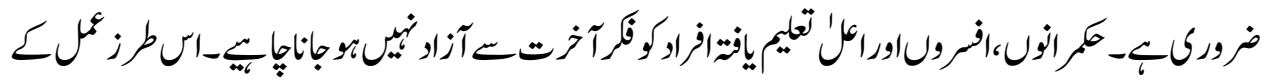

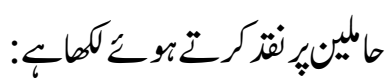

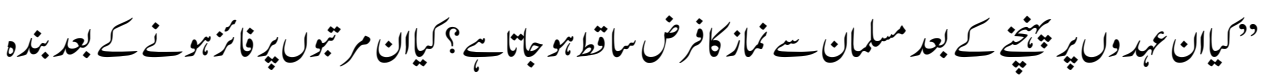

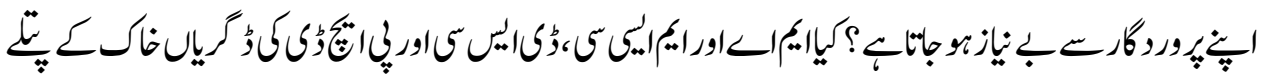

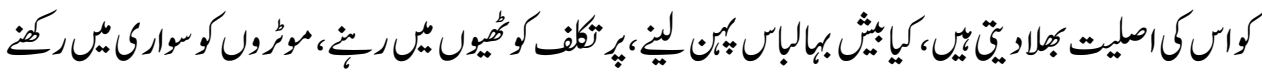

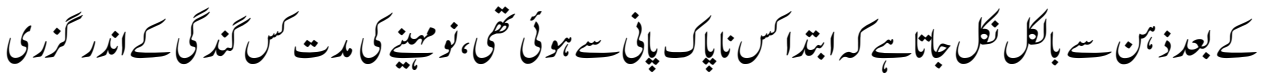

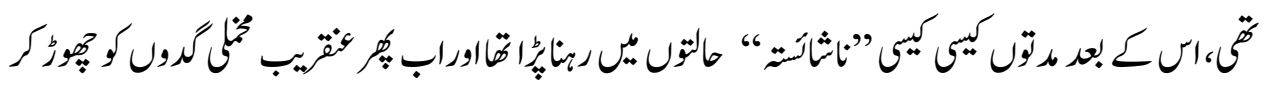

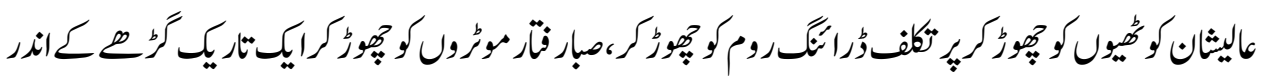

15 Millat e Islamia awr Asr e Hazir k Taqazay, P75

16 Ibid. p127 


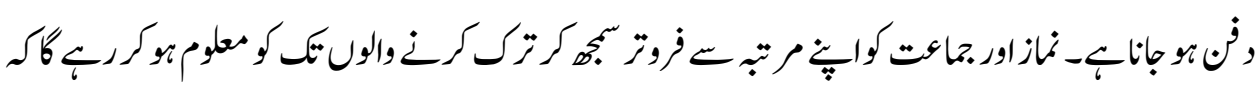

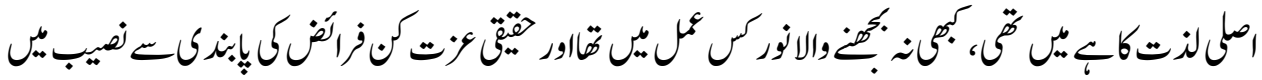

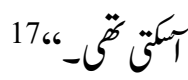

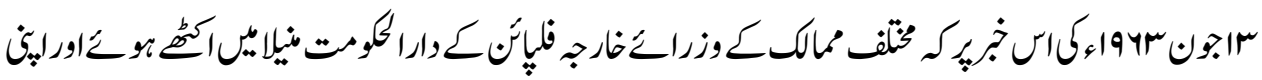

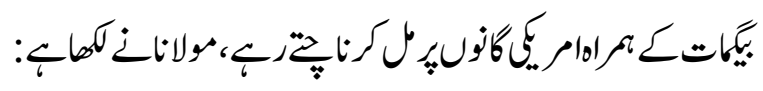

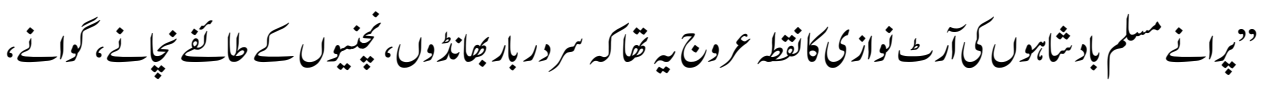

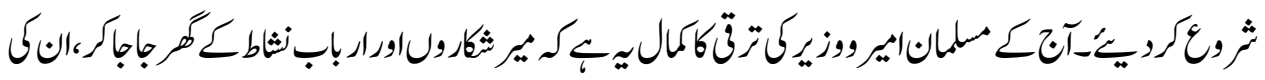

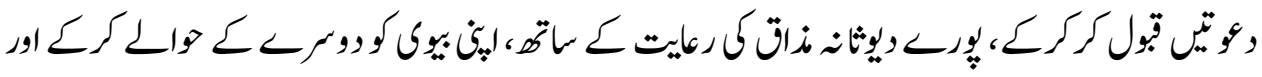

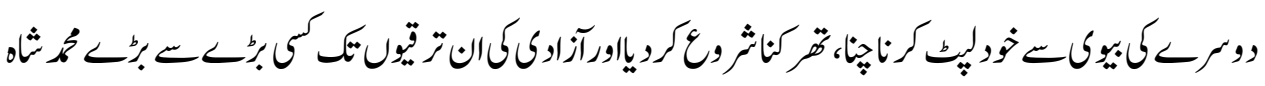

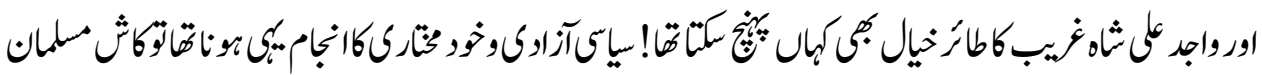

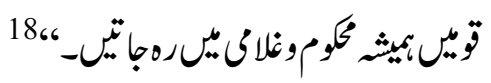

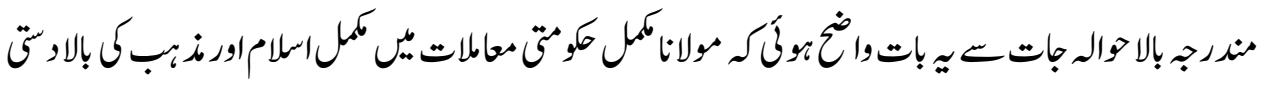

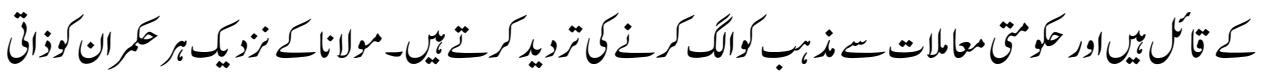

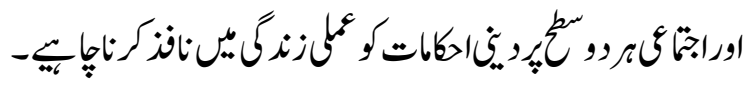

\section{تهنيبوتمكاناسلاماورمولاناريإبإك:}

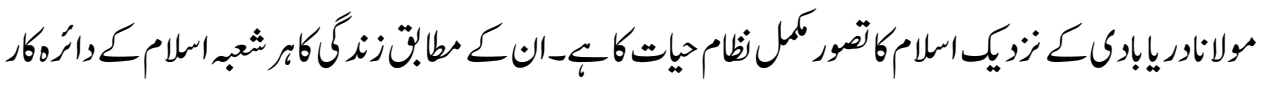

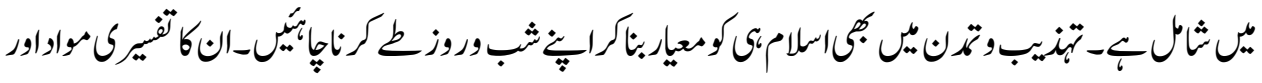

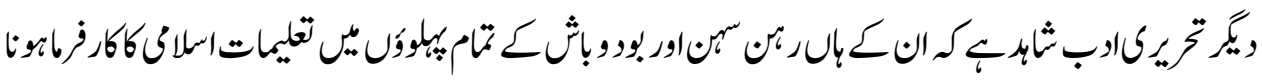

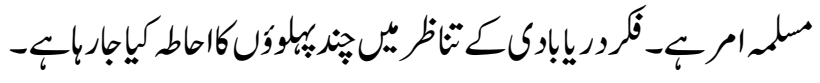
الكلوثرباوراسلاكهاليات:

${ }^{17}$ Ibid. p 114-115

18 Daryabadi, Abdul Majid, Maulana, "Islam, Musalman awr Tehzib e Jadid" Mashmula, Mujalla "Baidari” Mahnama, Haidar Abad, JildSoam, Shumara 22, January 2005, p 10 


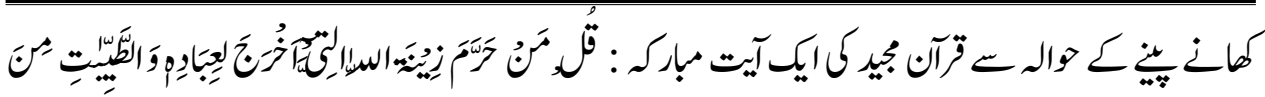

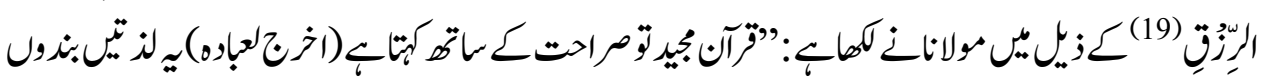

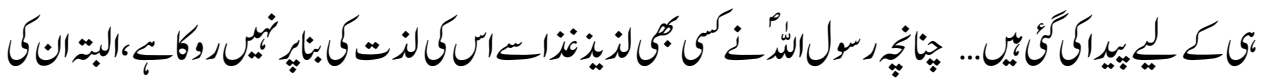

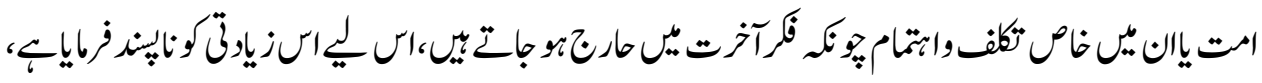

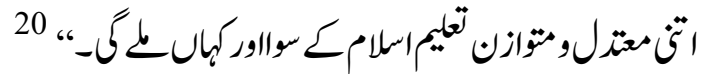

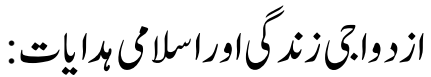

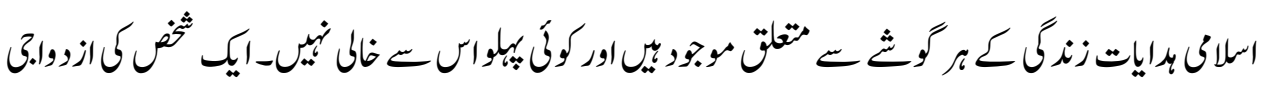

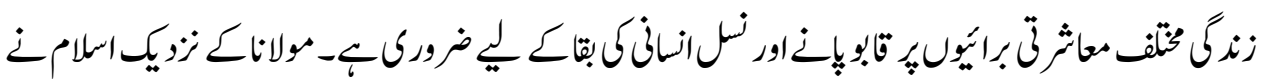

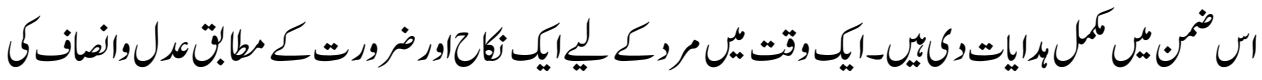

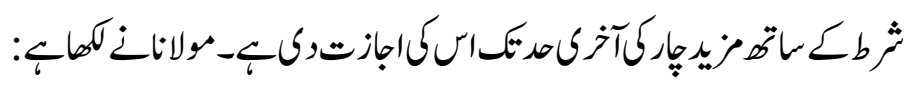

Monogamy is thus the ideal; and polygamy is only allowed as a "

safeguard against greater social evils." ${ }^{(21)}$

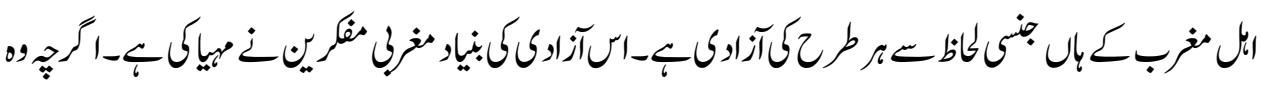

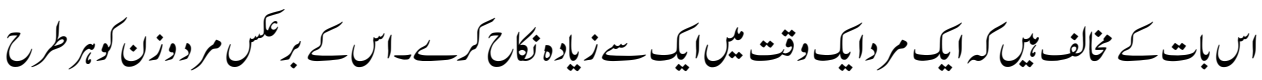

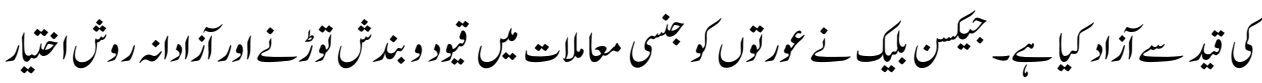

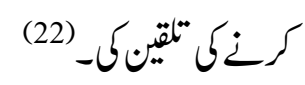

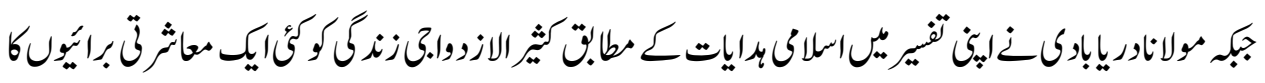

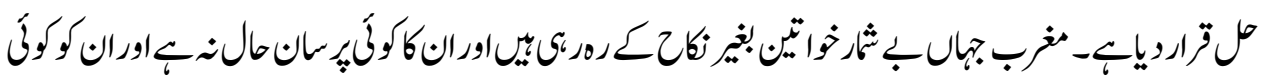

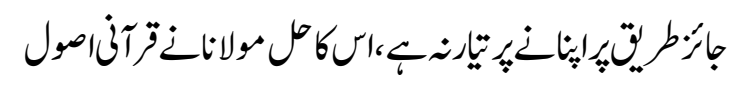

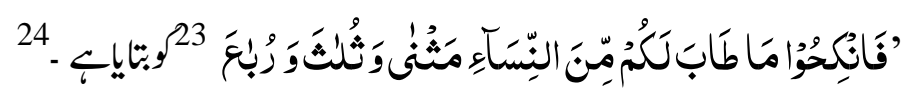

\footnotetext{
${ }^{19}$ Al-Araaf: 32

${ }^{20}$ Millat e Islamiah awr Asr e Hazir k Taqazay, p 70-71

21 Tafsir-e-Majidi, Vol I, P IV, n. 501

${ }^{22}$ Maghrabi Falsafa taleem aik Tanqidi Mutala'a, p53

${ }^{23}$ Al-Nisa: 3
} 


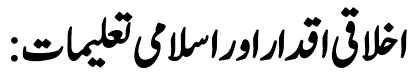

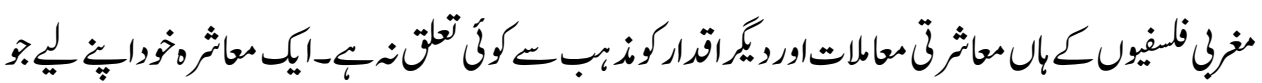

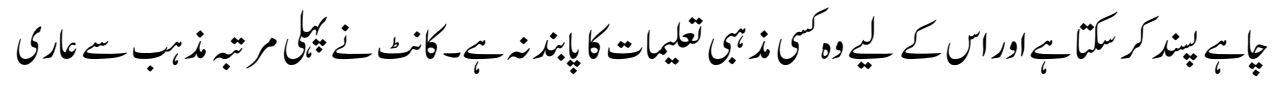

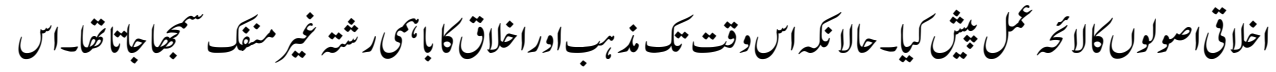

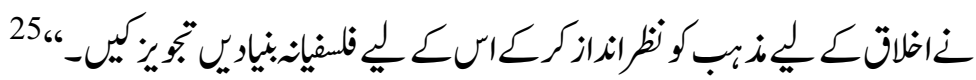

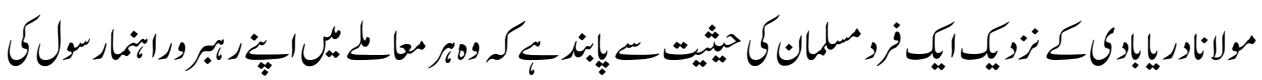

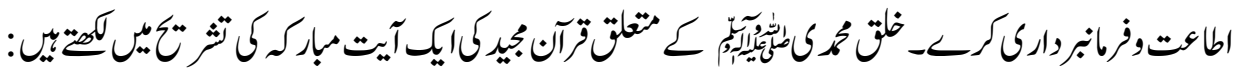
Thou standest on an exalted standard of character, and thy life is a " wonderful living illustration and explanation of the Quran itself." 26

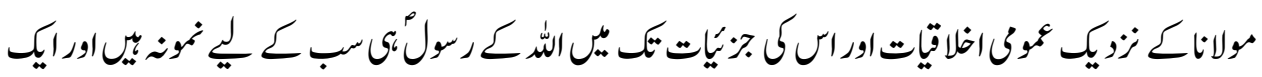

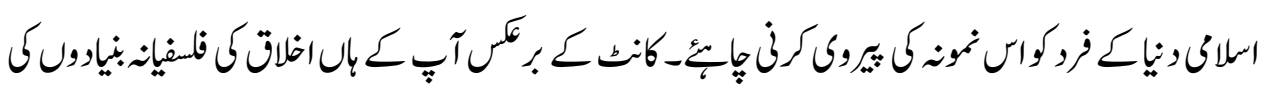

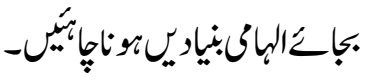

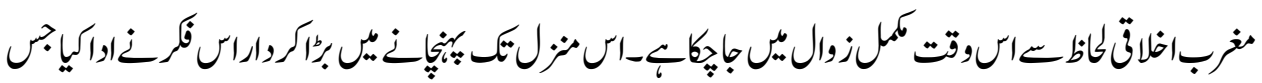

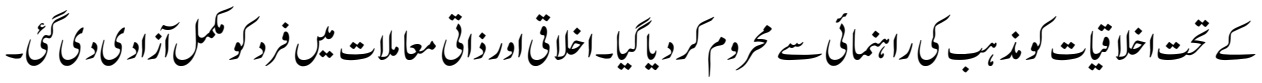

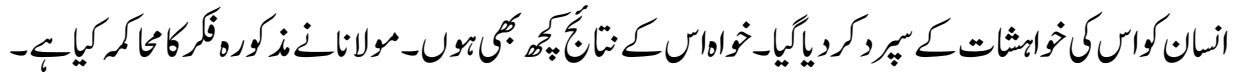

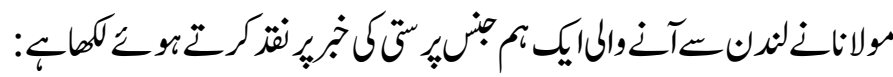

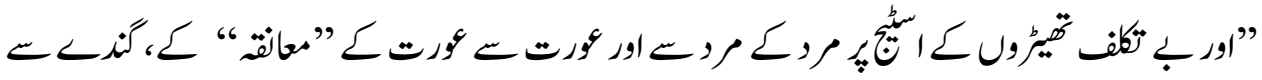

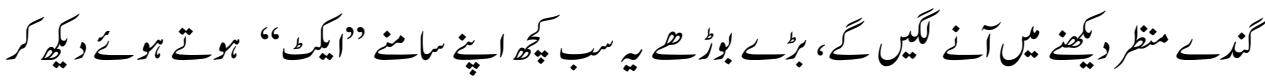

24 Tafsir-e-Majidi, Vol I, P IV, n 497

${ }^{25}$ Daryabadi, Abdul Majid, Maulana, Aalm e Islam, Dajjali Tehzib ki Zad main,

Murattab: Muhammad Musa Bhutto, Haidarabad: Sindh National Academy, 2006, p. 113

${ }^{26}$ Tafsir-e-Majidi, V IV, P XXIX, n 68 
The Scholar Islamic Academic Research Journal

Vol. 6, No. 1 || January-June 2020 || P. 41-55

https://doi.org/10.29370/siarj/issue10ar5

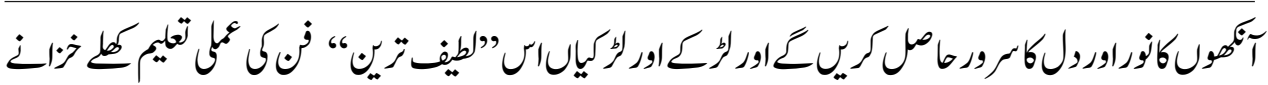

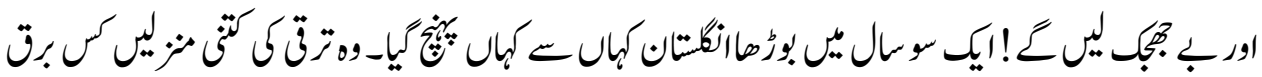

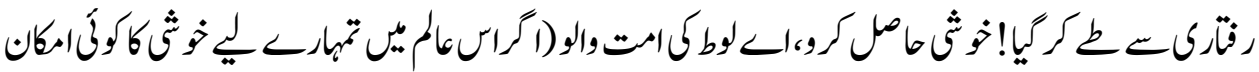

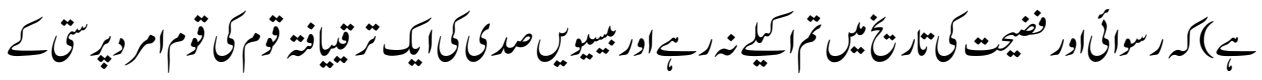

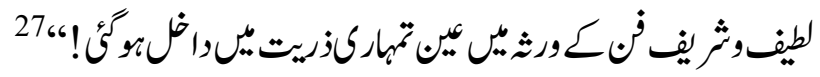

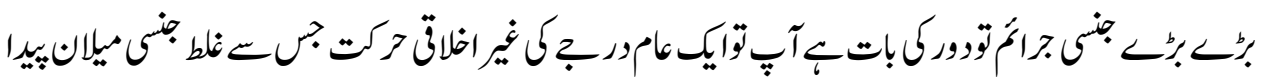

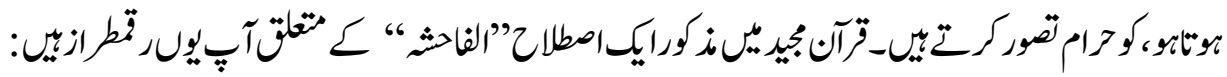
in the context does not signify the act of lewdness but الفاحش The word" the dissemination of scandalous news and gossip, the wide-spread social vice ...... the word of scandal 'repeated and reiterated at hundreds of luncheon tables, tea tables, dinner tables, bridge tables and supper tables." ${ }^{28}$

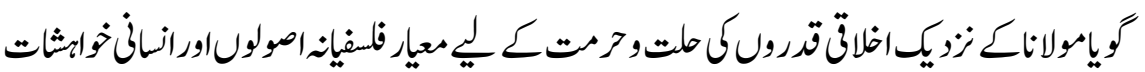

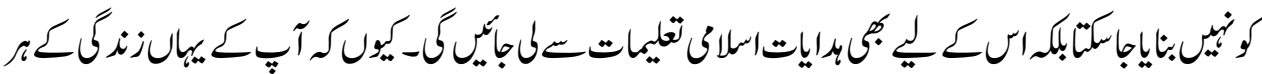

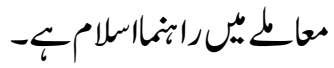

\section{سيك رلتيم اور مولاناوريابادى:}

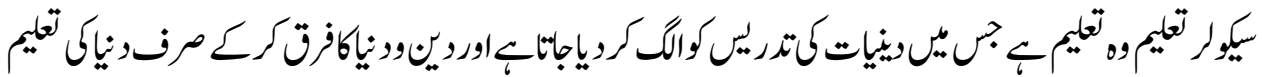

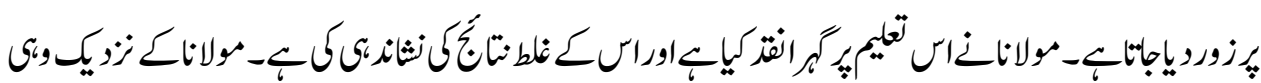

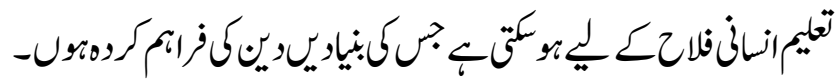
طبقات كمواضح تقنيم:

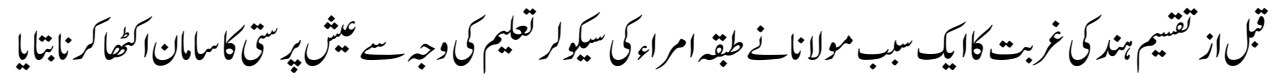

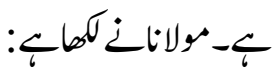

27 Aalm e Islam, Dajjali Tehzib ki Zad main, p.242

28 Tafsir-e-Majidi, V III, P XVIII, n. 217 


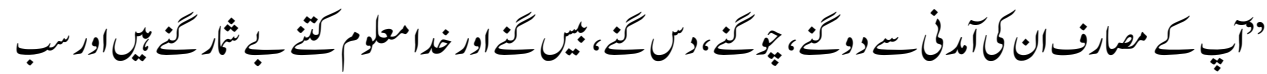

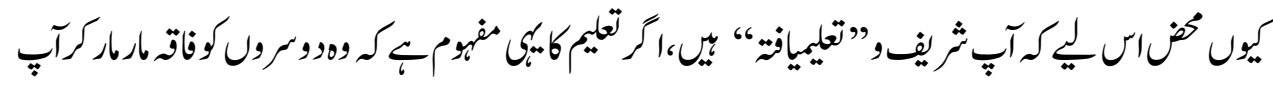

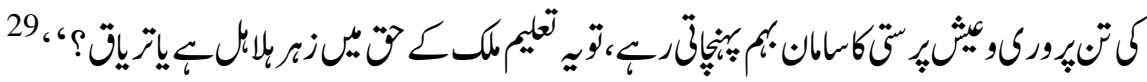

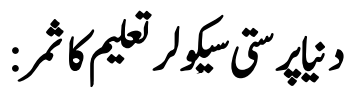

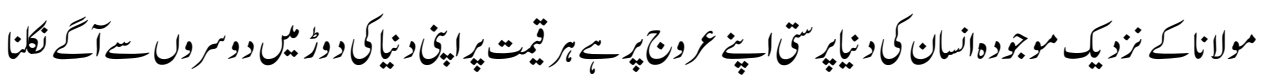

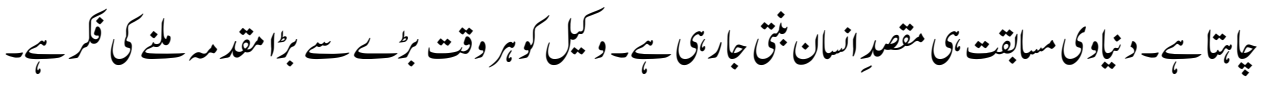

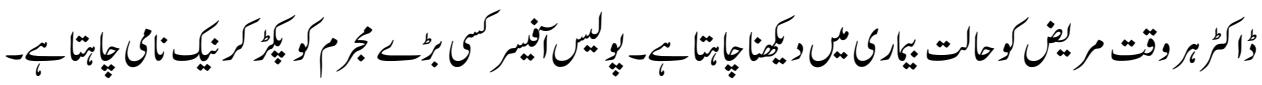

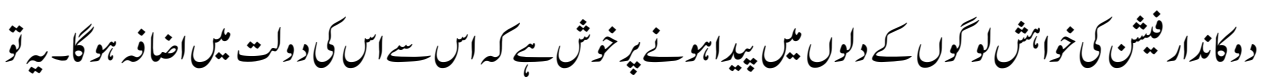

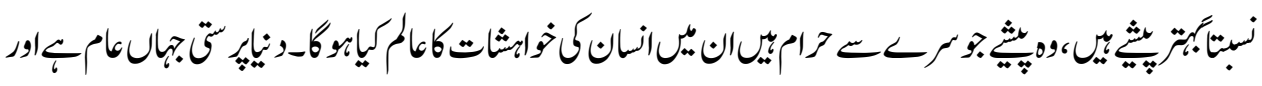

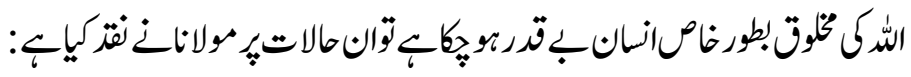

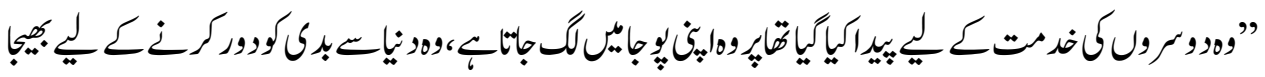

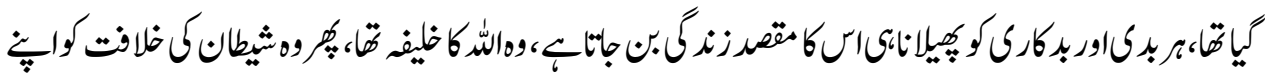

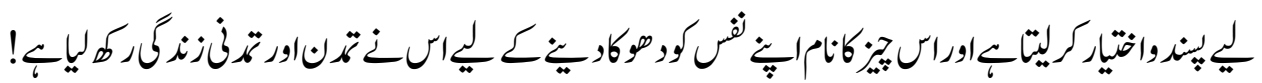

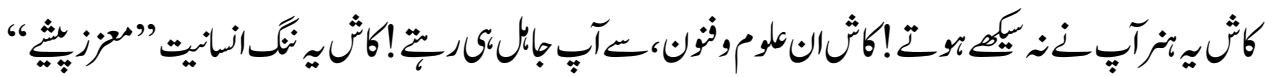

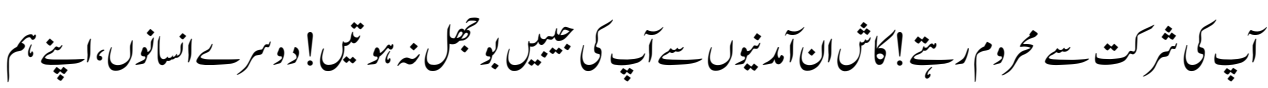

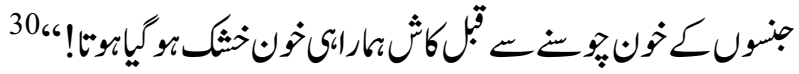

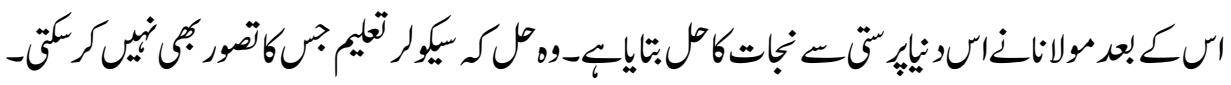

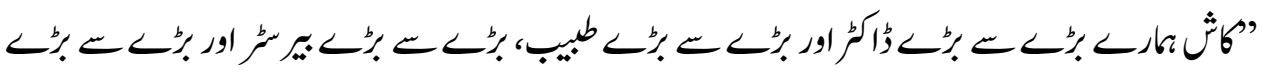

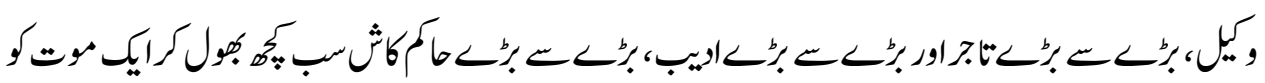
يابركت_-31،

${ }^{29}$ Millat e Islamia oar Asr e Hazir k taqazay, p.242

30 Ibid. p.242

31 Ibid. 


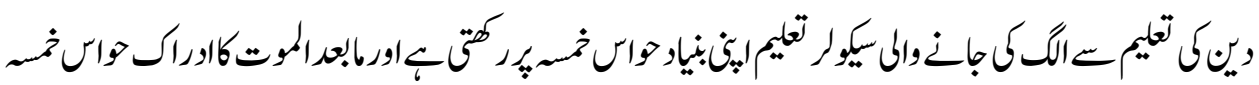

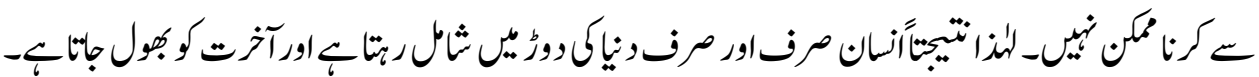

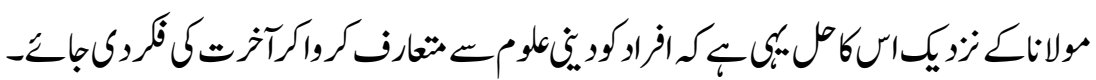

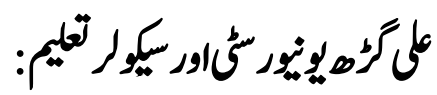

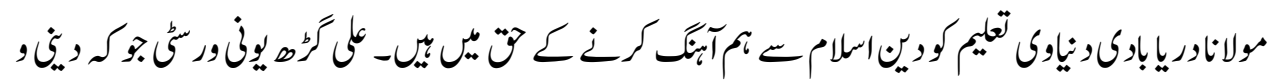

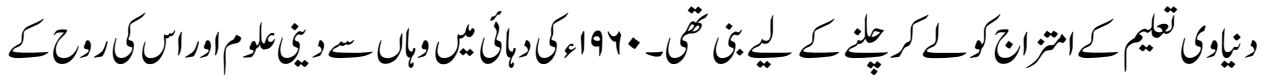

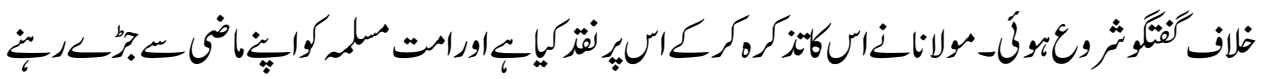

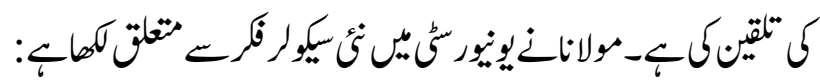

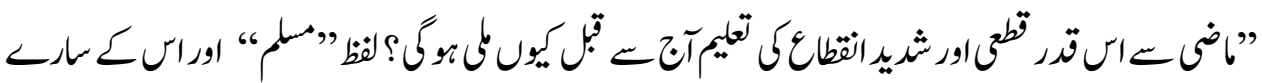

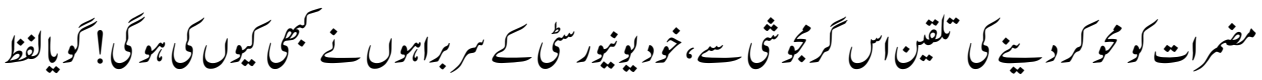

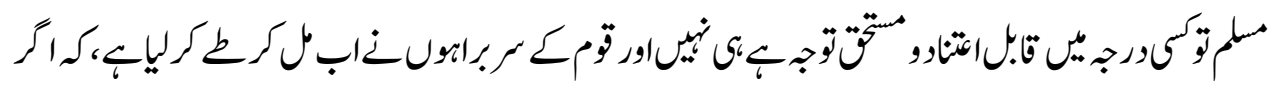

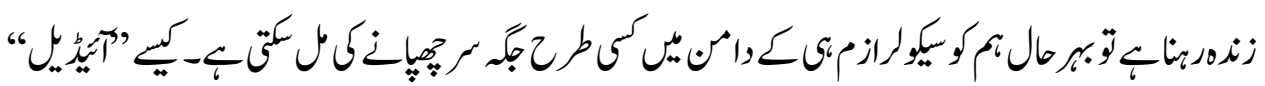

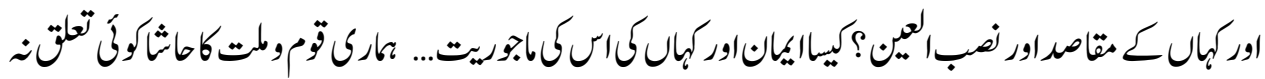

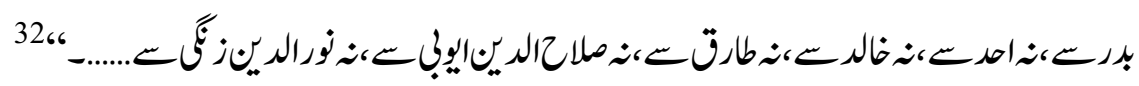

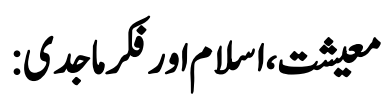
مخرف فرك:

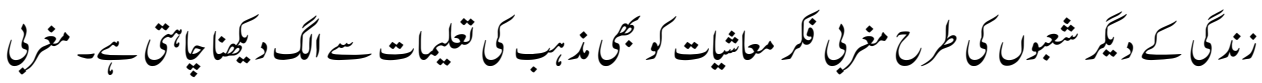

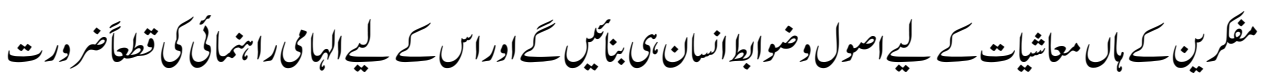

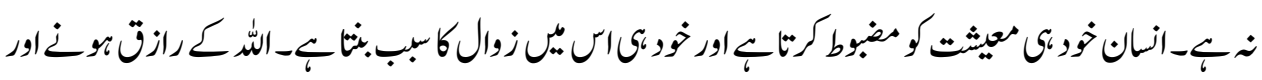

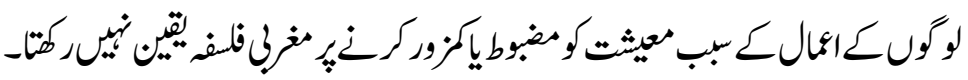

${ }^{32}$ Ibid. p124-125 


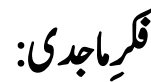

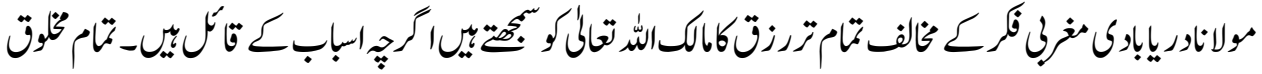

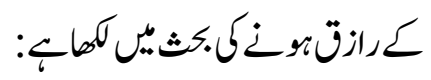

Providing every living creature with its food is incumbent upon the " great Provider." 33

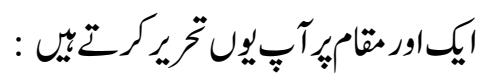

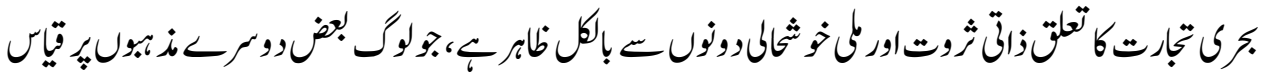

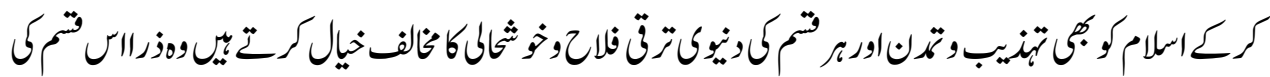

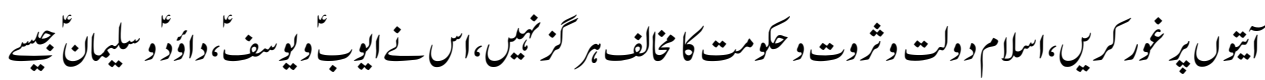

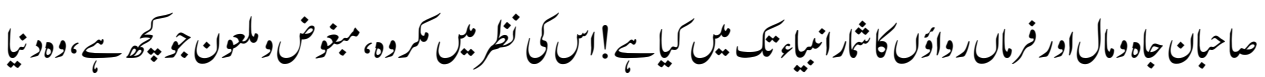

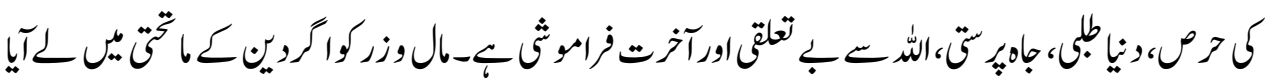

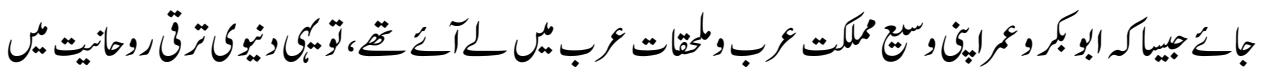

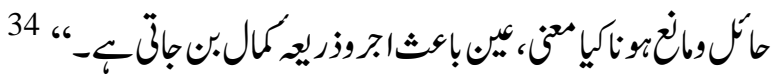

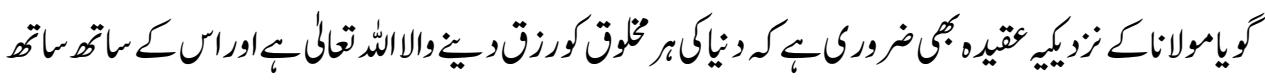

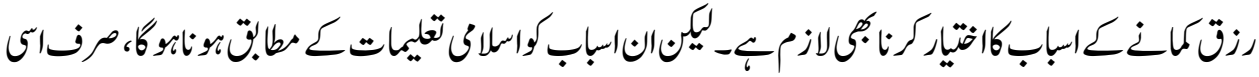

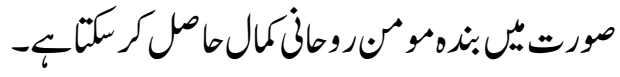




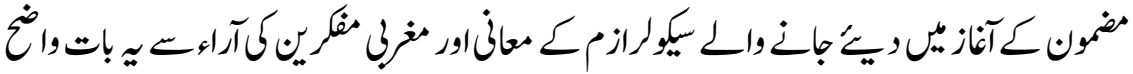

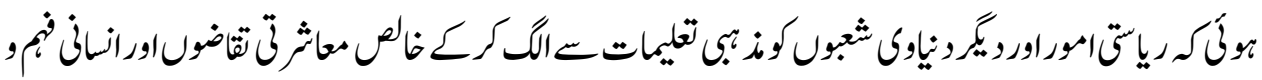

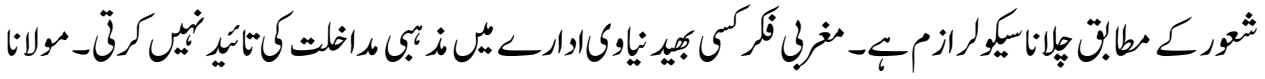

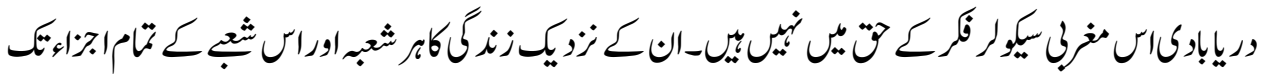

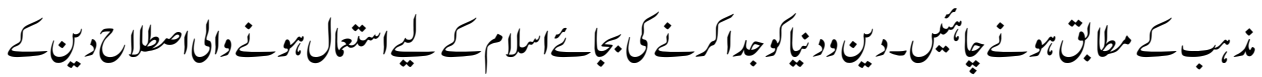

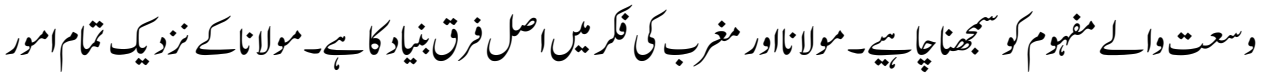

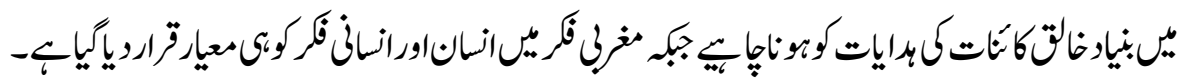

